\title{
The conformational changes of Zika virus methyltransferase upon converting SAM to SAH
}

\author{
Han Zhou ${ }^{1,2, *}$, Fenghua Wang ${ }^{1, *}$, Haofeng Wang ${ }^{1, *}$, Cheng Chen ${ }^{1}$, Tianqing Zhang ${ }^{1}$, \\ Xu Han ${ }^{1}$, Deping Wang ${ }^{1}$, Chen Chen ${ }^{1}$, Chen Wu ${ }^{1}$, Wei $X^{1}{ }^{1}{ }^{1}$, Zefang Wang ${ }^{1}$, Lei \\ Zhang $^{1}$, Lanfeng Wang ${ }^{3}$, Haitao Yang ${ }^{1,2}$ \\ ${ }^{1}$ School of Life Sciences, Tianjin University, Tianjin 300072, China \\ ${ }^{2}$ Tianjin International Joint Academy of Biotechnology and Medicine, Tianjin 300457, China \\ ${ }^{3}$ Key Laboratory of Molecular Virology and Immunology, Institute Pasteur of Shanghai, Chinese Academy of Sciences, \\ Shanghai 200031, China \\ *These authors contributed equally to this work
}

Correspondence to: Haitao Yang, email: yanght@tju.edu.cn Lanfeng Wang, email: lanfwang@ips.ac.cn

Keywords: Zika virus, methyltransferase, crystal structure, SAH, antiviral drug development

Received: November 16, 2016

Accepted: January 11, 2017

Published: January 21, 2017

\section{ABSTRACT}

An outbreak of Zika virus (ZIKV) infection has been reported in South and Central America and the Caribbean. Neonatal microcephaly potentially associated with ZIKV infection has already caused a public health emergency of international concern. Currently, there are no clinically effective vaccines or antiviral drugs available to treat ZIKV infection. The methyltransferase domain (MTase) of ZIKV nonstructural protein 5 (NS5) can sequentially methylate guanine $\mathrm{N}-7$ and ribose $2^{\prime}-0$ to form ${ }^{\mathrm{m}}{ }^{\mathrm{N}} \mathrm{GpppA} \mathrm{2}^{2} \mathrm{Om}$ cap structure in the new RNA transcripts. This methylation step is crucial for ZIKV replication cycle and evading the host immune system, making it a target for drug design. Here, we present the $1.76 \AA$ crystal structure of ZIKV MTase in complex with the byproduct SAH, providing insight into the elegant methylation process, which will benefit the following antiviral drug development.

\section{INTRODUCTION}

Zika virus (ZIKV), a member of the Flavivirus genus in the family Flaviviridae, is transmitted primarily by Aedes mosquitoes. It has attracted the attention worldwide due to the severe threats to public health [1-6]. ZIKV infection during pregnancy may cause microcephaly in newborn infants [3-7] and is also considered as a trigger of Guillain-Barré syndrome [7, 8]. ZIKV is closely related to Dengue Virus, West Nile Virus, and other flaviviruses. There are no clinically effective vaccines or antiviral drugs available to treat ZIKV infection to this date.

ZIKV nonstructural protein 5 (NS5) is a multi-domain protein, which has an N-terminal methyltransferase domain (MTase) and a C-terminal RNA dependent RNA polymerase (RDRP) domain. The MTase can sequentially methylate guanine $\mathrm{N}-7$ and ribose $2^{\prime}-\mathrm{O}$ to form ${ }^{\mathrm{m} 7 \mathrm{~N}} \mathrm{GpppA}{ }^{2^{\prime} \mathrm{Om}}$ cap structure in the new RNA transcripts [9-11]. The methylation starts with transferring the methyl group from the donor S-adenosylmethionine (SAM) to the methyl acceptor and then leaves the
S-adenosylhomocysteine (SAH) as a byproduct (Figure 1A). This methylation step is crucial for ZIKV replication cycle and evading the host immune system $[12,13]$. Defects in MTase are lethal for flaviviruses, which makes MTase a potential target for rational drug design against flaviviruses. Although the crystal structure of ZIKV MTase bound to SAM and 7-methyl guanosine diphosphate (7-MeGpp) was recently published [14], the stepwise of catalytic mechanism is still largely unknown. Here, we present the $1.76 \AA$ crystal structure of ZIKV MTase in complex with the byproduct $\mathrm{SAH}$, providing insight into the elegant methylation process, which will benefit the following antiviral drug development.

\section{RESULTS}

The structure of ZIKV MTase in complex with SAH

The crystal structure of ZIKV MTase was determined at $1.76 \AA$ by molecular replacement using 
the structure of PDB ID: 5KQR [14] as the original search model with the $R_{\text {free }}$ of $19.9 \%$ and $R_{\text {work }}$ of $17.2 \%$ (Supplementary Table 1). The MTase forms a well-defined 3D structure consisting of nine $\alpha$-helixes (cyan, $\alpha 1-\alpha 9$ ), nine $\beta$-strands (magenta, $\beta 1-\beta 9$ ), and multiple loop regions. Together, the $\alpha 4-\alpha 8, \beta 2-\beta 8$, and interspersed loops form the Rossman fold, which is a protein structural motif that binds nucleotides (Figure 1B, Supplementary Figure 1). In this structure, the byproduct SAH binds in the pocket of the N-terminal portion of the Rossman fold, which is primarily surrounded by $\alpha 4-\alpha 6$, $\beta 2, \beta 3, \beta 5$, and adjacent loop regions. Clear electron density can be identified for SAH (Figure 1B and Supplementary Figure 2). In the complex structure, SAH is stabilized by hydrogen bond network formed between the ligand and the adjacent residues. Among them, H110, E111, V130, and D131 form the hydrogen bonds with the adenosyl group of SAH; S56, W87, and D146 form the hydrogen bonds with the homocysteine group of SAH (Figure 1C-1D). The ligand comes in contact with residues $\mathrm{K} 105, \mathrm{G} 106$, and $\mathrm{E} 111$ by ionic interactions. Furthermore, the side chains of F133, V132, and I147 can stack together with the base of SAH to enhance hydrophobic interactions (Figure 1C).
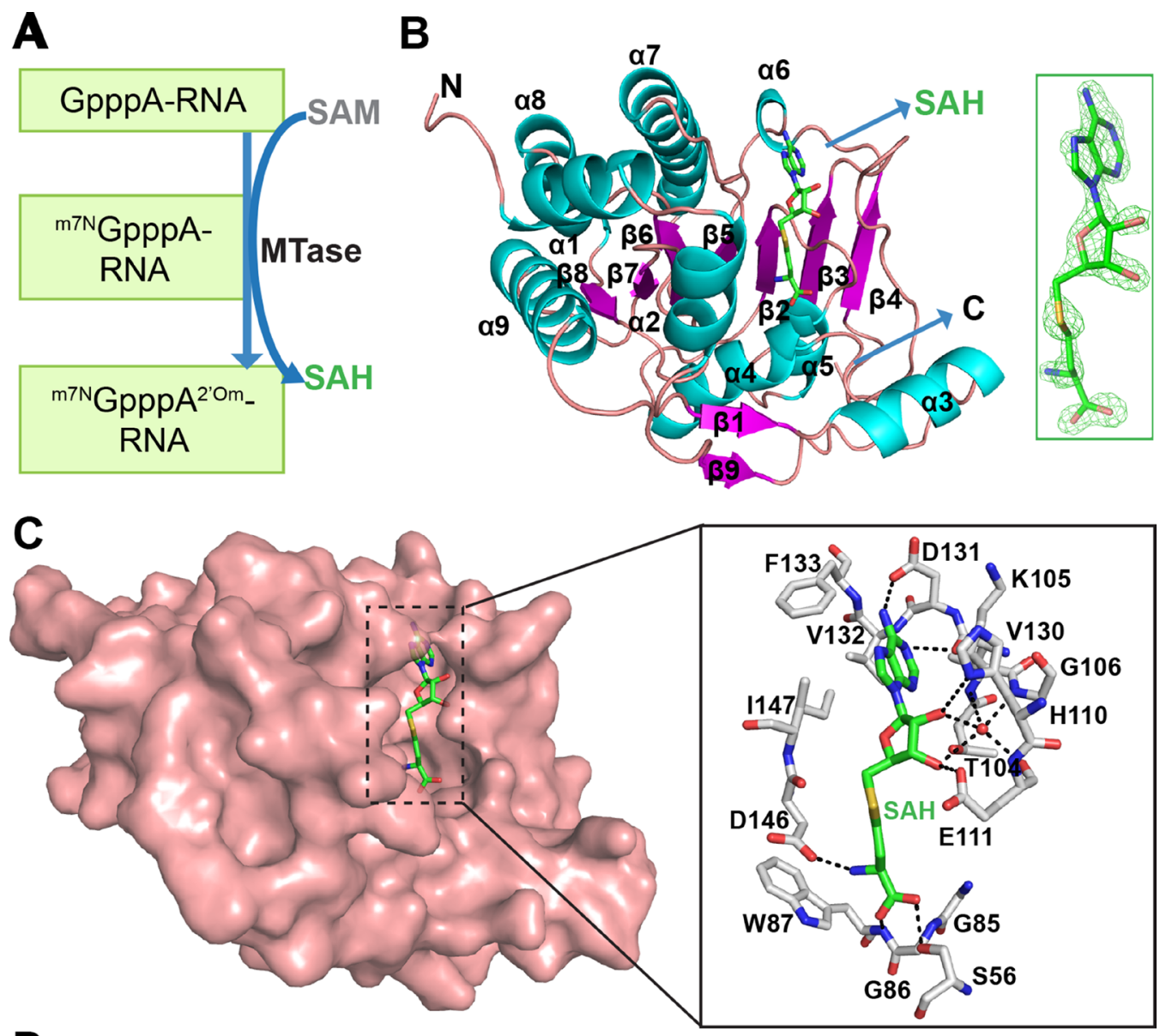

D

\begin{tabular}{|c|c|c|c|c|c|c|c|}
\hline $\begin{array}{c}\text { Atom/ } \\
\text { Residue }\end{array}$ & $\begin{array}{c}\text { OG/ } \\
\text { S56 }\end{array}$ & $\begin{array}{c}\text { N/ } \\
\text { W87 }\end{array}$ & $\begin{array}{c}\text { ND1/ } \\
\text { H110 }\end{array}$ & $\begin{array}{c}\text { OE1/ } \\
\text { E111 }\end{array}$ & $\begin{array}{c}\text { O/ } \\
\text { V130 }\end{array}$ & $\begin{array}{c}\text { OD1/ } \\
\text { D131 }\end{array}$ & $\begin{array}{c}\text { OD2/ } \\
\text { D146 }\end{array}$ \\
\hline $\begin{array}{c}\text { Atom/ } \\
\text { SAH }\end{array}$ & 01 & OXT1 & O2' & O3' & N1 & N6 & N1 \\
\hline $\begin{array}{c}\text { Distance/ } \\
(\mathrm{A})\end{array}$ & 2.7 & 3.2 & 3.7 & 2.7 & 3.3 & 3.0 & 2.8 \\
\hline
\end{tabular}

Figure 1: The structure of ZIKV MTase in complex with the byproduct SAH. (A) The steps in which MTase sequentially methylates guanine N-7 and ribose 2'-O to form the ${ }^{\mathrm{m} 7 \mathrm{~N}} \mathrm{GpppA}^{2 \prime \mathrm{Om}}$ RNA cap structure. (B) Ribbon representation of the overall structure of MTase in complex with SAH (Green), which was clearly defined by the omit electron density map contoured at 3.0 б. Cyan, helix; Magenta, strand; Salmon, loop. (C) The detailed interactions between SAH and MTase. (D) The distances are indicated for the hydrogen bonds in panel C. 


\section{The conformational changes of ZIKV MTase upon converting SAM to SAH}

In order to understand the stepwise catalytic mechanism for ZIKV MTase, we compared our structure with the published MTase structure in the presence of 7-MeGpp and SAM (Figure 2A). The overall structure of ZIKV MTase in complex with SAH is very similar with the reported one (PDB ID: 5KQS) with a RMSD of $0.242 \AA$ ( 205 out of $266 \mathrm{Cas}$ ). Meanwhile, the byproduct (SAH) was overlaid quite well with the substrate (SAM). However, we found that the size of the substrate/ byproduct-binding pocket decreases dramatically upon converting SAM to SAH. The binding pocket was closed mainly through two gatekeeper residues: E149 and H110. And the surface area of the binding pocket decreased from $907 \AA^{2}$ to $867 \AA^{2}$. Additionally, the top region of the binding pocket forms a defined $\alpha$ helix $(\alpha 6)$ instead of a random coil.

Interestingly, we noticed that the 7-MeGpp binding channel (Figure 2B) is significantly narrower in the presence than in the absence of 7-MeGpp. No obvious conformational changes were observed for the top region of the binding pocket, which is composed of the residues E149, S150, S151, and S152. However, the bottom region, which comprises residues M19, S20, A21, L22, E23 in the loop connecting $\alpha 1$ and $\alpha 2$, and residues F24, Y25, S26,
$\mathrm{K} 28$, and $\mathrm{K} 29$ in $\alpha 2$ helix have significant conformational changes in both the backbone and the side chains. The main chain moved as far as $1.1 \AA$ and caused the binding channel to shrink. Additionally, the residues ranging from 21 to 29 form a well-define $\alpha$ helix ( $\alpha 2)$ in our structure instead of a random coil in the published structure with 7-MeGpp (PDB ID: 5KQS).

Taken together, both the SAM/SAH binding pocket and the 7-MeGpp binding channel decreased in size upon converting SAM to SAH during catalysis.

\section{DISCUSSION}

ZIKV MTase structure is highly conserved in the genus Flavivirus. The comparison of our structure and those of other flavivirus MTases has shown that this protein is well conserved among the genus. The RMSD between the structure of ZIKV MTase and those of Dengue virus (for 230 Cas, PDB ID: 4V0Q [15]), yellow fever virus (for 227 Cas, PDB ID: 3EVA [16]) and West Nile virus (for $228 \mathrm{Cas}$, PDB ID: $2 \mathrm{OY0}$ [17]) are $0.52 \AA$, $0.47 \AA$ and $0.53 \AA$, respectively. The high homology of flavivirus MTases suggests that it could be a drug target to develop wide-spectrum antivirals.

The conformational changes of both SAM and 7-MeGpp binding sites of MTase upon converting SAM to SAH during catalysis has not been reported for any

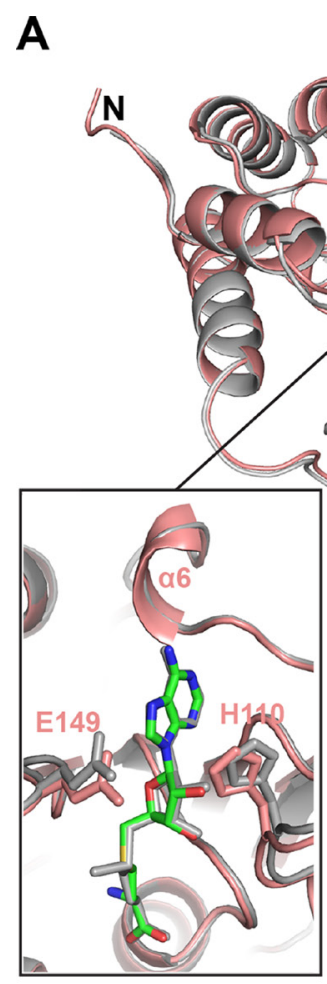

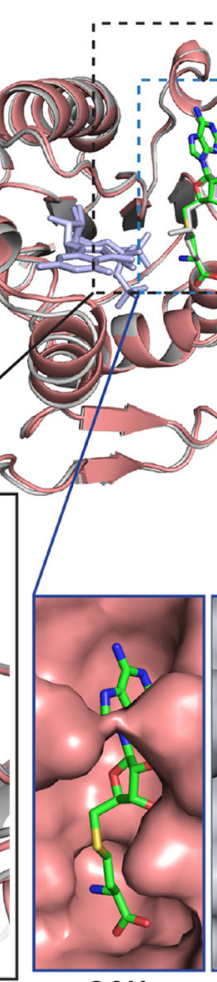

SAH
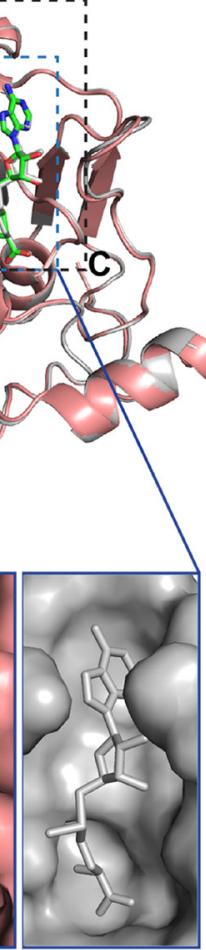

SAM

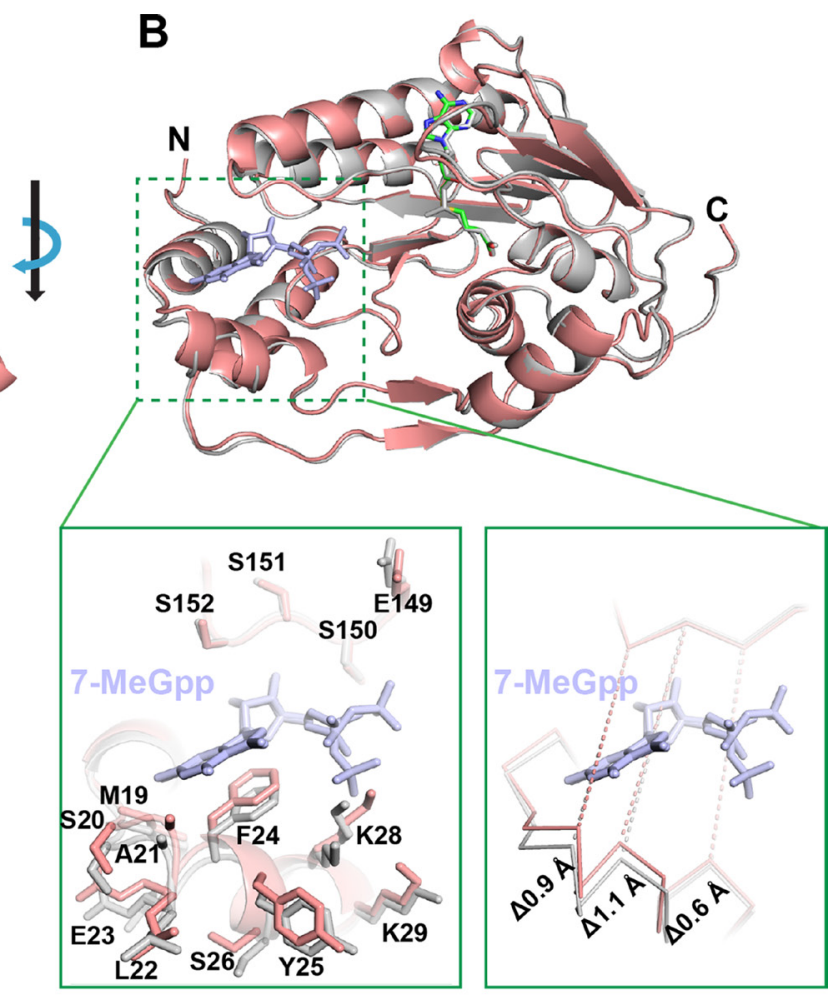

Figure 2: The conformational changes of ZIKV MTase upon converting SAM to SAH. (A) Comparison of the conformational change of the binding pocket of SAM with that of SAH. (B) Shrinking 7-MeGpp binding channel in the absence of 7-MeGpp in comparison with the one in the presence of 7-MeGpp. (Salmon, our structure; gray, PDB ID: 5KQS). 
flavivirus. In our study, the comparison of these structures at high resolution provides accurate details to elaborate the stepwise catalytic mechanism for flavivirus MTase, providing the structural basis for rational drug design against ZIKV or other flaviviruses. The future research will focus on the structures of MTase in complex with initial substrate GpppA-RNA, final product ${ }^{\mathrm{m} 7 \mathrm{~N}} \mathrm{GpppA}^{2^{\prime} \mathrm{Om}}$ RNA, and other transition state mimics to elucidate the RNA capping mechanisms, which is essential for the viral replication cycle and evading host immune system.

\section{MATERIALS AND METHODS}

\section{Protein expression and purification}

The ZIKV MTase (residues 1-266) coding fragment was inserted to the BamHI and XholI restriction sites of the pET28b-SUMO vector for expression in the Escherichia coli BL21 (DE3). Cells were grown in LB medium at $37^{\circ} \mathrm{C}$ and then induced by $0.5 \mathrm{mM}$ isopropyl- $\beta$-Dthiogalactopyranoside at $16^{\circ} \mathrm{C}$. The cells were harvested and resuspended in lysis buffer A (25 mM Tris- $\mathrm{HCl}, \mathrm{pH} 8.0$, $0.5 \mathrm{M} \mathrm{NaCl}, 5 \%$ glycerol and $2 \mathrm{mM} \beta$-mercaptoethanol) and lysed by high pressure homogenization. The supernatant after centrifugation was loaded onto the NiNTA column (GE). The column was washed using buffer A supplemented with $20 \mathrm{mM}$ imidazole and eluted using buffer A supplemented with $250 \mathrm{mM}$ imidazole. After concentration by ultrafiltration, protein sample was diluted into buffer B (50 mM HEPES, pH7.5, 0.5 M NaCl, 5\% glycerol), and the fraction containing (His) ${ }_{6}-\mathrm{SUMO}-\mathrm{ZIKV}$ MTase was cleaved with ULP protease at $4^{\circ} \mathrm{C}$ overnight. The protein sample was then re-loaded on the Ni-NTA column to remove the (His) ${ }_{6}-\mathrm{SUMO}$ and the ULP protease and further purified by sequential chromatography: cation exchange column (Hi Trap SP 5 ml, GE) and gel-filtration column (Superdex 75 10/300 GL, GE). MTase was finally concentrated to $10 \mathrm{mg} / \mathrm{ml}(0.33 \mathrm{mM})$ and mixed with 1.98 $\mathrm{mM}$ SAH prior to crystallization.

\section{Crystallization, data collection, and structure determination}

The crystals of ZIKV MTase were grown in the buffer $(0.1 \mathrm{M}$ sodium citrate tribasic dihydrate $\mathrm{pH} 5.5$, $22 \%(\mathrm{w} / \mathrm{v})$ polyethylene glycol 1000$)$ at $18^{\circ} \mathrm{C}$ using the microbatch-under-oil method. The crystals were cryoprotected by Parabar 10312 (previously known as Paratone oil). Diffraction data were collected at $100 \mathrm{~K}$ at the Shanghai Synchrotron Radiation Facility (SSRF) beamline BL18U at a wavelength of $0.97776 \AA$. Diffraction data were processed using $H K L 3000$ [18]. The structure was solved using PHENIX [19] software package by molecular replacement using the structure of PDB ID: 5KQR [14] as the original search model. Multiple rounds of model building in COOT [20] and refinement in PHENIX were performed and led to a final model with $R_{\text {work }}$ of $17.2 \%$ and $\mathrm{R}_{\text {free }}$ of $19.9 \%$. The Ramachandran plot generated by PHENIX software package showed that the residues located in the favored and allowed regions are $98.1 \%$ and $1.9 \%$ respectively. No residues are located in the disallowed regions. The data collection and refinement statistics are summarized in Supplementary Table 1.

\section{Structural analysis and illustrations}

COOT and PYMOL (The PYMOL Molecular Graphics System, Version 1.8 Schrödinger, LLC) were used for the structural analysis and illustration.

\section{Protein structure accession number}

The refined coordinates have been deposited in the PDB under accession number 5WXB.

\section{ACKNOWLEDGMENTS}

We would like to thank Wen Cui for data collection at beamline BL18U of the Shanghai Synchrotron Radiation Facility (SSRF).

\section{CONFLICTS OF INTEREST}

The authors declared that they have no conflicts of interest.

\section{FUNDING}

This work was supported by National Key Basic Research Program of China (973 program) (No. 2015CB859800), National Key Research Program of China (No. 2016YFD0500300), National Natural Science Foundation of China (No. 31528006) and the startup funds from Institute Pasteur of Shanghai, Chinese Academy of Sciences.

\section{Authors' contributions}

Han Zhou, Lanfeng Wang and Haitao Yang conceived and designed the experiments. Han Zhou, Fenghua Wang, Haofeng Wang, Tianqing Zhang, Xu Han, Deping Wang, Chen Chen, Wei Xie performed the experiments. Cheng Chen, Chen Wu, Zefang Wang, Lei Zhang analyzed the data. Han Zhou, Lanfeng Wang and Haitao Yang wrote the paper.

\section{REFERENCES}

1. Faria NR, Azevedo Rdo S, Kraemer MU, Souza R, Cunha MS, Hill SC, Theze J, Bonsall MB, Bowden TA, Rissanen I, Rocco IM, Nogueira JS, Maeda AY, et al. Zika 
virus in the Americas: Early epidemiological and genetic findings. Science. 2016; 352:345-9. doi: 10.1126/science. aaf5036.

2. Lancet T. Zika virus: a new global threat for 2016. Lancet. 2016; 387:96. doi: 10.1016/S0140-6736(16)00014-3.

3. Lazear HM, Diamond MS. Zika Virus: New Clinical Syndromes and Its Emergence in the Western Hemisphere. J Virol. 2016; 90:4864-7 5. doi: 10.1128/JVI.00252-16.

4. Martines RB, Bhatnagar J, de Oliveira Ramos AM, Davi HP, Iglezias SD, Kanamura CT, Keating MK, Hale G, SilvaFlannery L, Muehlenbachs A, Ritter J, Gary J, Rollin D, et al. Pathology of congenital Zika syndrome in Brazil: a case series. Lancet. 2016; 388:898-904. doi: 10.1016/S01406736(16)30883-2.

5. Petersen LR, Jamieson DJ, Powers AM, Honein MA. Zika Virus. New England Journal of Medicine. 2016; 374.

6. Rasmussen SA, Jamieson DJ, Honein MA, Petersen LR. Zika Virus and Birth Defects - Reviewing the Evidence for Causality. New England Journal of Medicine. 1969; 11. doi: 10.1056/NEJMsr1604338.

7. Ladhani SN, O'Connor C, Kirkbride H, Brooks T, Morgan D. Outbreak of Zika virus disease in the Americas and the association with microcephaly, congenital malformations and Guillain-Barré syndrome. Archives of Disease in Childhood. 2016; 101:600-2. doi: 10.1136/archdischild-2016-310590.

8. Watrin L, Ghawché F, Larre P, Neau JP, Mathis S, Fournier E. Guillain-Barré Syndrome (42 Cases) Occurring During a Zika Virus Outbreak in French Polynesia. Medicine. 2016; 95. doi: 10.1097/MD.0000000000003257.

9. Bollati M, Milani M, Mastrangelo E, Ricagno S, Tedeschi G, Nonnis S, Decroly E, Selisko B, De LX, Coutard B. Recognition of RNA cap in the Wesselsbron virus NS5 methyltransferase domain: implications for RNA-capping mechanisms in Flavivirus. Journal of Molecular Biology. 2008; 385:140-52. doi: 10.1016/j.jmb.2008.10.028.

10. Egloff MP, Decroly E, Malet H, Selisko B, Benarroch D, Ferron F, Canard B. Structural and functional analysis of methylation and 5'-RNA sequence requirements of short capped RNAs by the methyltransferase domain of dengue virus NS5. Journal of Molecular Biology. 2007; 372:723-36. doi: 10.1016/j.jmb.2007.07.005.

11. Ray D, Shah A, Tilgner M, Guo Y, Zhao Y, Dong H, Deas TS, Zhou Y, Li H, Shi PY. West Nile virus 5'-cap structure is formed by sequential guanine N-7 and ribose
2'-O methylations by nonstructural protein 5. Journal of Virology. 2006; 80:8362-70. doi: 10.1128/JVI.00814-06.

12. Dong H, Chang DC, Xie X, Toh YX, Chung KY, Zou G, Lescar J, Lim SP, Shi PY. Biochemical and genetic characterization of dengue virus methyltransferase. Virology. 2010; 405:568-78. doi: 10.1016/j.virol.2010.06.039.

13. Zhou Y, Ray D, Zhao Y, Dong H, Ren S, Li Z, Guo Y, Bernard KA, Shi PY, Li H. Structure and function of flavivirus NS5 methyltransferase. Journal of Virology. 2007; 81:3891-903. doi: 10.1128/JVI.02704-06.

14. Coloma J, Jain R, Rajashankar KR, Garcia-Sastre A, Aggarwal AK. Structures of NS5 Methyltransferase from Zika Virus. Cell Rep. 2016; 16:3097-102. doi: 10.1016/j. celrep.2016.08.091.

15. Zhao Y, Soh TS, Zheng J, Chan KW, Phoo WW, Lee CC, Tay MY, Swaminathan K, Cornvik TC, Lim SP, Shi PY, Lescar J, Vasudevan SG, et al. A crystal structure of the Dengue virus NS5 protein reveals a novel inter-domain interface essential for protein flexibility and virus replication. PLoS Pathog. 2015; 11:e1004682. doi: 10.1371/journal.ppat.1004682.

16. Geiss BJ, Thompson AA, Andrews AJ, Sons RL, Gari HH, Keenan SM, Peersen OB. Analysis of flavivirus NS5 methyltransferase cap binding. J Mol Biol. 2009; 385:1643-54. doi: 10.1016/j.jmb.2008.11.058.

17. Zhou Y, Ray D, Zhao Y, Dong H, Ren S, Li Z, Guo Y, Bernard KA, Shi PY, Li H. Structure and function of flavivirus NS5 methyltransferase. J Virol. 2007; 81:3891-903. doi: 10.1128/JVI.02704-06.

18. Otwinowski Z, Minor W. (1997). Processing of X-ray diffraction data collected in oscillation mode. Methods in Enzymology 276: Macromolecular Crystallography, part A, 307-326. Methods in Enzymology: Academic Press. 307-26.

19. Afonine PV, Grosse-Kunstleve RW, Echols N, Headd JJ, Moriarty NW, Mustyakimov M, Terwilliger TC, Urzhumtsev A, Zwart PH, Adams PD. Towards automated crystallographic structure refinement with phenix.refine. Acta Crystallographica Section D. 2012; 68:352-67. doi: 10.1107/S0907444912001308.

20. Emsley P, Cowtan K. Coot: model-building tools for molecular graphics. Acta Crystallographica Section D. 2004; 60:2126-32. doi: 10.1107/S0907444904019158. 